\title{
Matter Power Spectrum for Convex Quartessence
}

\author{
R. R. R. Reis $\dagger$, M. Makler $\dagger+$ and I. Waga $\dagger$ \\ † Universidade Federal do Rio de Janeiro, Instituto de Física, CEP 21941-972, Rio de \\ Janeiro, RJ, Brazil \\ ‡ Observatório Nacional - MCT, CEP 20921-400, Rio de Janeiro, RJ, Brazil
}

\begin{abstract}
.
The possibility of unifying dark-matter and dark-energy has recently attracted considerable interest. In this so called quartessence scenario, a single component is responsible for both the clustering of matter and the accelerated expansion of the universe. A model archetype for such scenario is provided by the Chaplygin gas. Although this model is in agreement with the data on the expansion history, problems arise in the power spectrum of density fluctuations for adiabatic perturbations. In this contribution we consider other quartessence models and confirm that instabilities and oscillations in the matter power spectrum are a characteristic of more generic quartessence models, namely those with a convex equation of state. We show that, as in the Chaplygin case, this kind of problem can be solved by considering intrinsic non-adiabatic perturbations such that, as an initial condition, the perturbed fluid is gradient pressure free. We also discuss how the problems of adiabatic quartessence can be circumvented by other types of equations of state.
\end{abstract}




\section{Introduction}

The twentieth century witnessed the establishment of a cosmological model consistent with a number of astronomical observations. In this standard model, the dynamics of the universe is governed by the local distribution of matter-energy, following Einstein's theory of gravitation. On large scales the universe is well described within a nearly flat Friedmann-Lemaitre-Robertson-Walker (FLRW) cosmological scenario, and is presently undergoing an accelerated expansion. Despite the success of this model, several key features of the universe remain unclear. For example, what constitutes the darkmatter (DM) that would trigger gravitational clustering, and the dark-energy (DE) that is supposed to power the accelerated expansion? Are these two dark components separate entities or different manifestations of a single matter-content?

Although there are some candidates for the DM from particle physics [1, there is yet no evidence for these particles in laboratory experiments. For the DE the situation is even more puzzling since there is no natural candidate from fundamental physics, although the cosmological constant and a dynamical scalar field are the most popular 2]. Thus, the current information about DE and DM comes solely from astronomical observations. From a simplicity viewpoint it is interesting to investigate the possibility of describing the phenomenology associated with both DE and DM through a single matter-component, unifying dark matter (UDM) — or simply quartessence — [ 3], reducing to one the unknown components of the material substratum of the universe.

A few candidates for such UDM appeared in the literature in recent years, the most popular being the generalized Chaplygin gas [4, [5], 6, 17, 8], in what we call the Quartessence Chaplygin Model (QCM). For this specific model, several confrontations with observational data were performed and constraints were set on the model parameters. The generalized Chaplygin gas (as quartessence) appears to be compatible with all available data regarding the expansion history (see e.g. ref. [9]). However, for adiabatic perturbations, it was shown that the mass power spectrum presents strong oscillations and instabilities, allowing only models very close to the $\Lambda$ CDM limit [10. This problem can be avoided if non-adiabatic perturbations with a specific initial condition $(\delta p=0)$ are considered [11]. In [10] it was argued that these oscillations and instabilities would be present in any quartessence model such that $p=p(\rho)$. We argue that this is indeed the case for convex equations of state. This is illustrated through the investigation of two extreme models of convex quartessence, one with a very steep and another with a very gentle equation of state. We show that for these models, as in the QCM case, the above mentioned problems in the mass power spectrum can always be avoided with the same kind of entropy perturbation. On the other hand, we argue that the problems of adiabatic quartessence could be avoided by other types of equations of state.

The paper is organized as follows. In section 2 we review the QCM and introduce the two mentioned extreme quartessence models. The treatment of linear perturbations

in these models is discussed in section 3. The computation of the power spectrum 
in the adiabatic and nonadiabatic cases and comparison with observational results are developed in section 4. Finally, we sum up our results and present concluding remarks in section 5

\section{Phenomenological Models of Quartessence}

In this work we model the UDM component as a fluid with isotropic energy-momentum tensor, whose only independent components are the pressure $p$ and the density $\rho$. We further assume that the space averaged dynamical variables are related by the equation of state $(\mathrm{EOS}) \bar{p}=\bar{p}(\bar{\rho})$, i.e. the background is a perfect fluid. For this EOS to be a quartessence candidate, it has to fulfill some conditions. For example, neglecting the contribution of baryons, in the FLRW model, for the universe to undergo acceleration, one must have at present $(\bar{\rho}+3 \bar{p})<0$. Thus we require $\bar{p}$ to be sufficiently negative when $\bar{\rho}$ is small. On the other hand, in the past, for the UDM component to behave like dark matter we must have $\bar{\rho} \gg \bar{p}$, such that the universe was decelerating and the density scaled as $a^{-3}$ (where $a$ is the scale factor). A remarkable property of quartessence is that the weak energy condition is never violated in such models. From the energy conservation equation

$$
\dot{\bar{\rho}}=-(\bar{\rho}+\bar{p}) 3 \frac{\dot{a}}{a}=-\bar{\rho}(1+w(\bar{\rho})) 3 \frac{\dot{a}}{a} .
$$

it follows that the line $\bar{p}=-\bar{\rho}$ cannot be crossed. Since at early times we should have $w \approx 0$, a straightforward prediction of these models is, therefore, that $w>-1$. If observations find that $w<-1$, this automatically rules out quartessence models in which the background is a prefect fluid (i.e. in which $w$ is a function only of $\bar{\rho}$ ) and where the dark sector is not coupled to other components.

A simple example of an equation of state satisfying the above conditions is given by an inverse power law [4, [5], [6, 7, 8],

$$
\bar{p}=-\frac{M^{4(\alpha+1)}}{\bar{\rho}^{\alpha}} \quad \text { (Chaplygin Quartessence), }
$$

where $M$ has dimension of mass and $\alpha$ is a dimensionless parameter. The energy conservation equation (11) has a simple analytic solution for this EOS

$$
\bar{\rho}_{\mathrm{Ch}}=\bar{\rho}_{\mathrm{Ch} 0}\left[(1-A)\left(\frac{a_{0}}{a}\right)^{3(\alpha+1)}+A\right]^{1 /(\alpha+1)} .
$$

Here $a_{0}$ is the present value of the scale factor and $A=\left(M^{4} / \bar{\rho}_{\mathrm{Ch} 0}\right)^{(\alpha+1)}$. As expected, when $a / a_{0} \ll 1$, we have $\bar{\rho}_{\mathrm{Ch}} \propto a^{-3}$ and the fluid behaves as CDM. For late times, $a / a_{0} \gg 1$, and we get $\bar{p}_{\mathrm{Ch}}=-\bar{\rho}_{\mathrm{Ch}}=-M^{4}=$ const. as in the cosmological constant case.

The QCM has been extensively discussed in the literature recently, both setting observational limits to the model and studying its properties and physical motivation. Nevertheless, since the QCM is a prototypical and particularly simple example of quartessence we shall briefly review a few results concerning the parameters of the 
model. This will help to fix the notation and gain some intuition, as several aspects are similar in generic models of quartessence.

Since in QCM the equation of state parameter $w$ is a monotonic decreasing function, it is easy to see from equation (10) that the equation of state goes asymptotically to a cosmological constant form $\bar{p}(\bar{\rho})=-\bar{\rho}$. The minimum value of the density, obtained from the solution of this equality, will be denoted by $\bar{\rho}_{\text {min }}$ and is simply given by $M^{4}$ in the QCM. Naturally, the current density $\bar{\rho}_{\mathrm{Ch} 0}$ cannot be lower than $\rho_{\text {min }}$ (otherwise, as the $\bar{p}=-\bar{\rho}$ line cannot be crossed, QCM would always have had $w<-1$, never acting as dark-matter). Thus $\bar{\rho}_{\text {min }}<\bar{\rho}_{\text {Ch0 }}<\infty$, which restricts the parameter $A$ to the range $0<a<1$. Clearly for $A=0$ the fluid behaves as dust, while for $A=1$ it acts as a cosmological constant.

Now, let us discuss the range of allowed values for $\alpha$. In most of the analyses in the literature it is assumed that $0 \leq \alpha \leq 1$. These limits are imposed because adiabatic perturbations of the QCM are implicitly assumed. Notice that, for these models, there is an absolute maximum value for the adiabatic sound speed $c_{s}^{2}=(\partial \bar{p} / \partial \bar{\rho})$, which occurs for $\bar{\rho}=\bar{\rho}_{\text {min }}$ and is given by $c_{s \max }^{2}=\alpha$. Therefore, to avoid superluminal propagation of signals the upper limit $(\alpha \leq 1)$ is imposed in the adiabatic case. Also, demanding stability of adiabatic perturbations implies $(\partial \bar{p} / \partial \bar{\rho}) \geq 0$, which requires $\alpha \geq 0$. However, as shown by Sandvik et al. [10], adiabatic QCM is ruled out by current data unless $\alpha$ is very close to zero (corresponding to the $\Lambda$ CDM limit). Nevertheless, if entropy perturbations such that the effective sound speed vanishes are allowed, the model is again consistent with observational data [11]. In this nonadiabatic case, the above limits on $\alpha$ are unnecessary. For instance, if $\alpha>1$ there is no problem of superluminal propagation for these specific entropy perturbations and even models with $\alpha<0$ can, in principle, be in accordance with observations. Since for quartessence we should have $w \approx 0$ as $\bar{\rho} \rightarrow \infty$, values of $\alpha<-1$ are not allowed. Therefore, here we only require that $\alpha>-1$.

The QCM power spectrum exhibits strong oscillations or instabilities, when adiabatic perturbations are considered and $\alpha$ is not very close to zero. As we shall see in the next section, this is due to the development in the fluid of a non null adiabatic sound speed at recent times. For QCM the adiabatic sound speed is related to the equation of state parameter by $c_{s}^{2}=\alpha\left(M^{4} / \bar{\rho}\right)^{(\alpha+1)}=-\alpha w$. To avoid such oscillations in linear scales, the parameter $\alpha$ of the QCM has to be restricted to $|\alpha|<10^{-5}$, very close to the $\Lambda C D M$ limit 10. One may wonder if a steeper variation of the equation of state would reduce the oscillations, allowing a wider range of parameters, distinct from the $\Lambda \mathrm{CDM}$ model. Let us consider for example an exponential equation of state

$$
\bar{p}=-M^{4} \exp \left(-\frac{\alpha \bar{\rho}}{M^{4}}\right) \quad \text { (Exponential Quartessence) }
$$

In this case, the adiabatic sound speed has an additional exponential suppression, $c_{s}^{2}=\alpha \exp \left(-\alpha \bar{\rho} / M^{4}\right)$. Thus, one could expect that high ratios of $\bar{\rho} / M^{4}$ would attenuate the oscillations in the power spectrum, without imposing strong constraints on the parameter $\alpha$. However, the equation of state parameter $w$ also has the same additional 
exponential factor and, if the ratio $\bar{\rho} / M^{4}$ is always high, the Universe does not enter in an accelerated phase. Therefore, as it will be shown explicitly in the next section, this modification in the equation of state does not help in solving the problem and indicates that it is a general property of the model.

Before introducing our third type of quartessence, let us discuss some noteworthy values of the exponential model parameters. First, for Eq. (41) to be a quartessence candidate $(w \approx 0$ as $\bar{\rho} \rightarrow \infty)$ the parameter $\alpha$ must be non negative. The condition $\alpha \geq 0$ also guarantees stability for adiabatic fluctuations. The minimum energy density of this model is given by $\bar{\rho}_{\text {min }}=M^{4} W(\alpha) / \alpha$, where $W(x)$ is the Lambert function defined by $W(x) \exp (W(x))=x$ [12]. Therefore, the maximum adiabatic sound speed is $c_{s \text { max }}^{2}=W(\alpha)$.

Another extreme case, such that the model has a $\Lambda$ CDM limit, is given by:

$$
\bar{p}=-\frac{M^{4}}{\left[\ln \left(\bar{\rho} / M^{4}\right)\right]^{\alpha}} \quad \text { (Logarithmic Quartessence) }
$$

In this case, for $w<0$ to be an increasing function of $\bar{\rho}$, either $\alpha \geq 0$ or $\alpha<-e$. The minimum density is given by $\bar{\rho}_{\text {min }}=M^{4} \exp \left(\alpha W\left(\alpha^{-1}\right)\right)$ and $c_{s \max }^{2}=1 / W\left(\alpha^{-1}\right)$. As in the exponential and QCM cases, for $\alpha=0$ we recover the $\Lambda$ CDM model for the background.

In the adiabatic case, where the condition $\alpha>0$ is fulfilled such that $c_{s}^{2}>0$, the three models discussed above have a common property, namely that the equation of state is convex, i.e. $d^{2} p / d \rho^{2}=d c_{s}^{2} / d \rho<0$. Therefore, the maximum value of the sound speed is reached at the minimum value of the density. We shall refer to models that share this property as convex quartessence. The above new Anzätze (Eqs. 4 and 5) cover two extreme cases of convex quartessence equations of state, one with a very steep and other with a very gentle variation of the pressure with the density. We believe that by analyzing these two examples we may have indications of generic behaviors for any (monotonic, well behaved, smooth, etc.) convex quartessence EOS. In the next sections we shall focus on the matter power spectrum of these models, show that they have the same oscillations as the Chaplygin case and check that the introduction of nonadiabatic fluctuations solves this problem.

\section{Evolution of Linear Perturbations}

The relativistic equations that govern the linear evolution of scalar perturbations in a multi-component fluid, in the synchronous gauge, are [13, 14]

$$
\begin{aligned}
\delta_{i}^{\prime}+3\left(c_{s i}^{2}-w_{i}\right) \frac{a^{\prime}}{a} \delta_{i} & =-\left(1+w_{i}\right)\left(k v_{i}+\frac{h_{L}^{\prime}}{2}\right)-3 w_{i} \frac{a^{\prime}}{a} \Gamma_{i}, \\
v_{i}^{\prime}+\left(1-3 c_{s i}^{2}\right) \frac{a^{\prime}}{a} v_{i} & =\frac{c_{s i}^{2}}{1+w_{i}} k \delta_{i}+\frac{w_{i}}{1+w_{i}} k \Gamma_{i}, \\
h_{L}^{\prime \prime}+\frac{a^{\prime}}{a} h_{L}^{\prime} & =-\sum_{i}\left(1+3 c_{s i}^{2}\right) 8 \pi G \bar{\rho}_{i} a^{2} \delta_{i}-24 \pi G a^{2} \sum_{i} \bar{p}_{i} \Gamma_{i},
\end{aligned}
$$


where $\delta_{i}, v_{i}$, and $\Gamma_{i}$ are, respectively, the density contrast, the velocity perturbation, and the entropy perturbation of component $i, h_{L}$ is the trace of the metric perturbation, and $k$ is the commoving wave number. For the sake of simplicity, we assumed that both the spatial curvature and the anisotropic pressure perturbation vanish and that the energy-momentum tensor of each component is separately conserved. In the equations above the prime denotes derivative with respect to conformal time $(d t=a d \eta)$ and, as usual, $c_{s i}^{2}=\bar{p}_{i}^{\prime} / \bar{\rho}_{i}^{\prime}$ and $w_{i}=\bar{p}_{i} / \bar{\rho}_{i}$ are, respectively, the adiabatic sound speed and the equation of state parameter of component $i$.

We will consider only two components in the universe: baryons $\left(\bar{p}_{b}=0\right)$ and a quartessence component. To glimpse general features of quartessence, we consider the two extreme models introduced above: exponential (Eq. 4) and the logarithmic quartessence (Eq. 5). As remarked, these models have the $\Lambda$ CDM model as a limiting case for the background when $\alpha=0$. At first and higher orders in perturbation theory, however, these quartessence models with $\alpha=0$, have distinct behavior as compared to that of dark-matter in $\Lambda$ CDM [15, 16].

The independent conservation of energy-momentum for baryons and quartessence leads to

$$
\bar{\rho}_{b}=\bar{\rho}_{b 0} a^{-3} \text {. }
$$

For the exponential and logarithmic cases, the energy conservation equation does not have an analytical solution. We rewrite Eq. (11) as

$$
\frac{d u}{d a}+3 \frac{u}{a}(1+w)=0
$$

were $u=\bar{\rho} / \bar{\rho}_{0}, w=w(u, \alpha, \tilde{A})$, and $\tilde{A}=M^{4} / \bar{\rho}_{0}$. For the exponential case one has

$$
w=-\frac{\tilde{A}}{u} \exp \left(-\frac{\alpha u}{\tilde{A}}\right)
$$

whereas the logarithmic case leads to

$$
w=-\frac{\tilde{A}}{u}\left(\ln \frac{u}{\tilde{A}}\right)^{-\alpha} .
$$

Therefore, given $\alpha$ and $\tilde{A}$ we numerically solve (10) with the initial condition $u(1)=1$ to get $u(a)$ and then $\bar{\rho}=\overline{\rho_{0}} u$. Notice that, for each $\alpha, \tilde{A}$ has to be chosen such that the condition $0 \leq \bar{\rho}_{\min } / \rho_{0} \leq 1$ is fulfilled.

Let us write Eqs. (6] for the baryon-quartessence system. For baryons, as $c_{s b}^{2}=0$ and $w_{b}=0$, Eq. (7) implies $v_{b} \propto a^{-1}$. As a matter of simplification, we take $v_{b}=0$. Now Eq (6) implies $h_{L}^{\prime} / 2=-\delta_{b}^{\prime}$. Using this result in Eq. (8) and changing the "time" variable, we obtain, for the flat case $\left(\Omega_{b 0}+\Omega_{q 0}=1\right)$,

$$
\frac{d^{2} \delta_{b}}{d a^{2}}+\left(\frac{2}{a}+\frac{\ddot{a}}{\dot{a}^{2}}\right) \frac{d \delta_{b}}{d a}=\frac{3 H_{0}^{2}}{2 \dot{a}^{2}}\left\{\Omega_{b 0} a^{-3} \delta_{b}+\left(1-\Omega_{b 0}\right) u\left[\left(1+3 c_{s q}^{2}\right) \delta_{q}+3 w_{q} \Gamma_{q}\right]\right\} .
$$

For the quartessence component, Eq. (6) can be rewritten as

$$
\frac{d \delta_{q}}{d a}+3\left(c_{s q}^{2}-w_{q}\right) \frac{\delta_{q}}{a}=-\left(1+w_{q}\right)\left(\frac{k v_{q}}{\dot{a} a}-\frac{d \delta_{b}}{d a}\right)-3 \frac{w_{q}}{a} \Gamma_{q},
$$


whereas Eq. (7) gives

$$
\frac{d v_{q}}{d a}+\left(1-3 c_{s q}^{2}\right) \frac{v_{q}}{a}=\frac{k}{1+w_{q}} \frac{1}{a \dot{a}}\left(c_{s q}^{2} \delta_{q}+w_{q} \Gamma_{q}\right)
$$

The derivatives of the scale factor with respect to $t$ are given by

$$
\begin{aligned}
& \dot{a}=H_{0} \sqrt{\frac{\Omega_{b 0}}{a}+\left(1-\Omega_{b 0}\right) u a^{2}}, \\
& \ddot{a}=-\frac{H_{0}^{2}}{2}\left(\frac{\Omega_{b 0}}{a^{2}}+\left(1-\Omega_{b 0}\right) u\left(1+3 w_{q}\right) a\right) .
\end{aligned}
$$

In the next section we apply these results to compute the matter power spectrum for quartessence models.

\section{The Power Spectrum}

In order to obtain power spectra for the considered models we evolved Eqs. (13) 15) from $z=500$ to $z=0$, using the solutions of Eq. (10). At $z=500$ the quartessence fluid behaves like CDM and, to take this into account, proper initial conditions were established: we assumed $v_{q}=0$, a scale invariant primordial spectrum, and used the BBKS transfer function [17, with the following effective shape parameter [18, 19],

$$
\Gamma_{e f f}=\left(\Omega_{b 0}+\Omega_{m}^{e f f}\right) h \exp \left(-\Omega_{b 0}-\frac{\sqrt{2 h} \Omega_{b 0}}{\Omega_{b 0}+\Omega_{m}^{e f f}}\right),
$$

where the term $\Omega_{m}^{e f f}=\left(1-\Omega_{b 0}\right) \lim _{a \rightarrow 0}\left(u a^{3}\right)$ is the effective matter density.

In Fig. 11 we present the baryon and the total mass power spectra, for the exponential quartessence model, in the adiabatic case $\left(\Gamma_{q}=0\right)$, for $\alpha=$ $0,10^{-5}, 10^{-4}, 10^{-3}$ (from top to bottom), $h=0.7, \Omega_{b 0}=0.04$, and we choose $M^{4} / \bar{\rho}_{0}$ such that all models have $\Gamma_{\text {eff }}=0.18$. The total power spectrum can be written as $P(k) \propto\left|\Omega_{b} \delta_{b}+\Omega_{q} \delta_{q}\right|^{2}$. The normalization of the mass power spectra is arbitrarily fixed at $k=0.01 \mathrm{~h} \mathrm{Mpc}^{-1}$. The (blue) squares are data points from the Sloan Digital Sky Survey (SDSS) as compiled in [20], whereas the (red) circles correspond to the Two Degree Field Galaxy Redshift Survey (2dFGRS) from [21. The presence of oscillations in the total power spectra, due to a non-vanishing adiabatic sound speed of the quartessence component, is apparent in the figure. Further, even for the baryonic component, the spectrum is compatible with the data only for $\alpha \ll 10^{-5}$. In Fig. 2, we display the baryon and total mass power spectra for logarithmic quartessence, for the same parameter values as in Fig. 11 Again, oscillations and power suppression are present in the total power spectrum. However, for $\alpha \lesssim 10^{-4}$, the baryonic power spectrum is still compatible with the data.

Notice that the amplitude of the initial power spectrum could be reescaled such that the baryon spectrum provides a better fit to the galaxy data. However, the normalization of the total power spectrum is constrained by gravitational lensing observations. Therefore, as remarked in [10, it is not possible to reconcile the constraints 

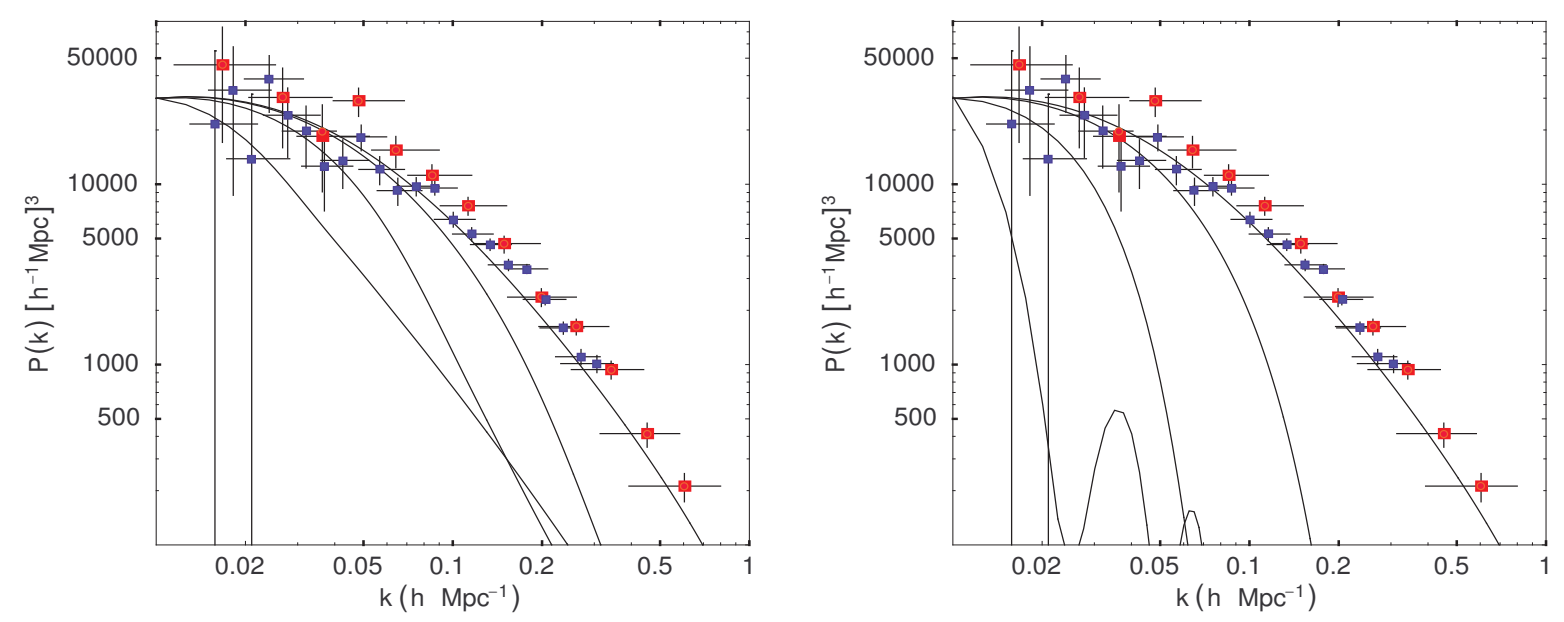

Figure 1. Baryon (left panel) and total (right panel) mass power spectra for exponential quartessence, in the adiabatic case. The curves from top to bottom correspond to $\alpha=0,10^{-5}, 10^{-4}, 10^{-3}$ and $M^{4} / \bar{\rho}_{0}$ is chosen such that all models have $\Gamma_{\text {eff }}=0.18$. The (blue) squares are the data points from the Sloan Digital Sky Survey as compiled in 20] and the (red) circles are from the $2 \mathrm{dF}$ galaxy redshift survey as compiled in 21].
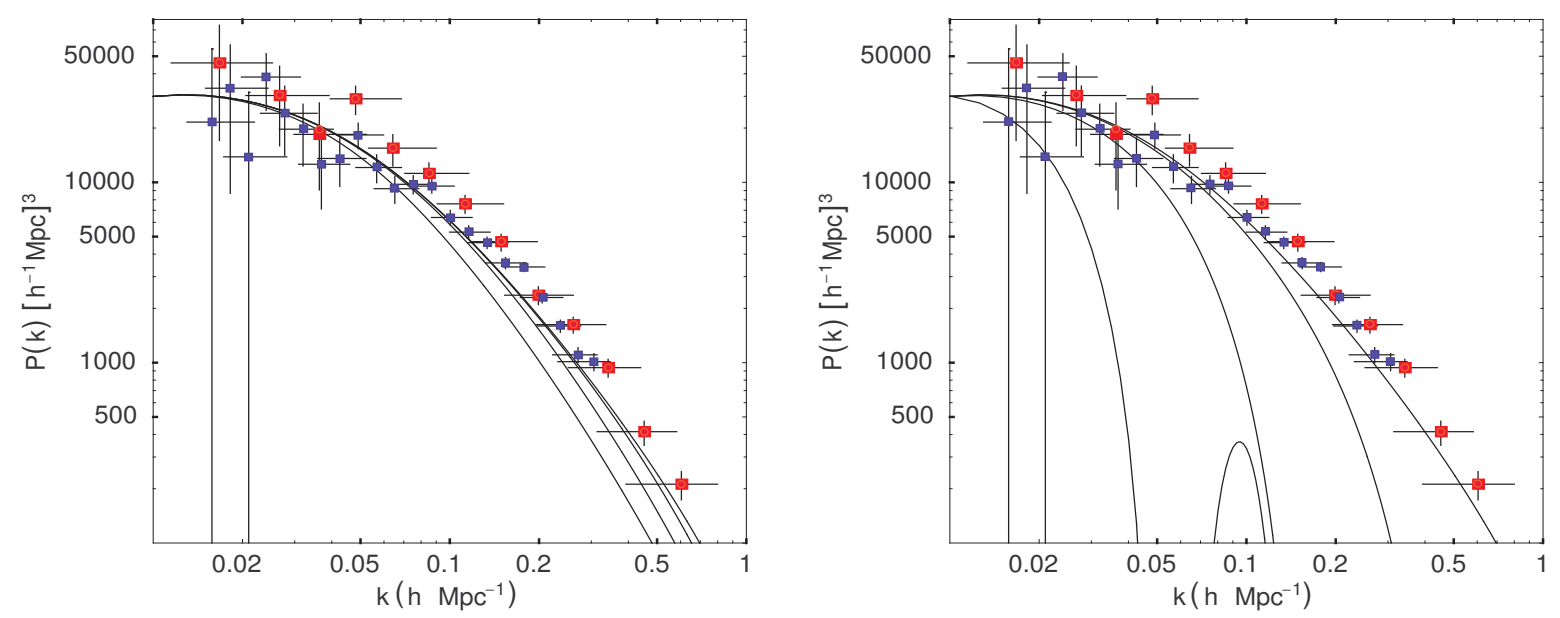

Figure 2. Baryon (left panel) and total (right panel) mass power spectra for logarithmic quartessence, in the adiabatic case. The curves from top to bottom correspond to $\alpha=0,10^{-5}, 10^{-4}, 10^{-3}$ and $M^{4} / \bar{\rho}_{0}$ is chosen such that all models have $\Gamma_{\text {eff }}=0.18$. The (blue) squares are the data points from the Sloan Digital Sky Survey as compiled in [20] and the (red) circles are from the $2 \mathrm{dF}$ galaxy redshift survey as compiled in 21]. 

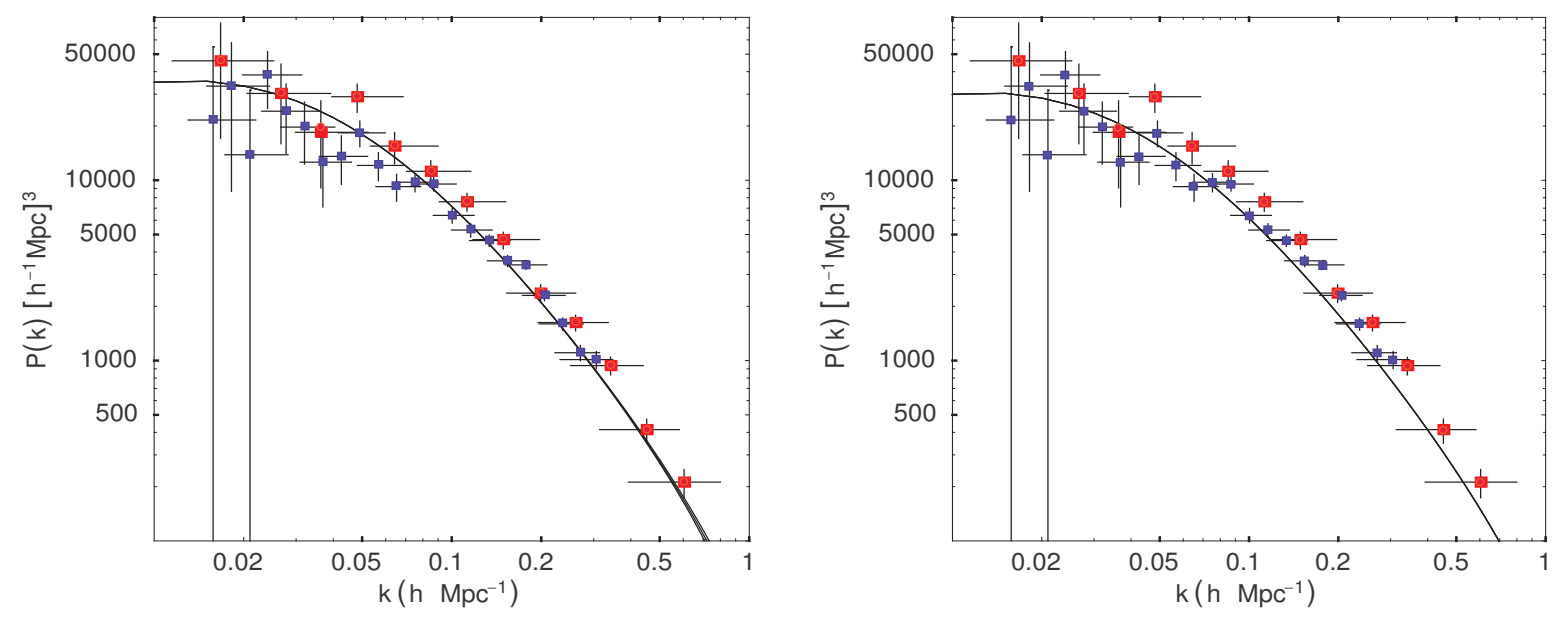

Figure 3. Baryon mass power spectra for the model with exponential quartessence (left panel) and logarithmic quartessence (right panel), in the non-adiabatic case, $\alpha=0,0.1,0.2$ and $M^{4} / \bar{\rho}_{0}$ chosen such that all models have $\Gamma_{\text {eff }}=0.18$. The (blue) squares are the data points from the Sloan Digital Sky Survey as compiled in [20] and the (red) circles are from the $2 \mathrm{dF}$ galaxy redshift survey as compiled in 21].

from both the galaxy power spectrum and gravitational lensing observations for non negligible values of $\alpha$ and adiabatic perturbations.

As discussed in [11 the oscillations (for $c_{s}^{2}>0$ ) and instabilities (for $c_{s}^{2}<0$ ) in the quartessence mass power spectrum that are present in the adiabatic case, have their origin in a non-vanishing value of the right-hand side in Eq. (17). To avoid these effects we choose, for the quartessence component

$$
\Gamma_{q}=-\frac{c_{s q}^{2}}{w_{q}} \delta_{q}=-c_{s q}^{2} \frac{\delta \rho_{q}}{p_{q}} .
$$

¿From the definition of entropy perturbation [13], we have

$$
\delta p=p \Gamma+c_{s}^{2} \delta \rho .
$$

Thus the condition (19) is equivalent to set $\delta p_{q}=0$. Moreover, it is sufficient that relation (19) be satisfied at some initial time $t$, since this condition is preserved along the linear evolution. This can be easily seen by differentiating (20) with respect to time and substituting (19), which implies that $d \delta p_{q} / d t=0$, with no consideration about the exact form of the fluid equation of state.

In Fig. 3 we show the baryon spectrum for exponential and logarithmic quartessence, in the case $\Gamma_{q}=-c_{s q}^{2} \delta_{q} / w_{q}$, for the same parameter values of Figs. [1 and 2. The results are visually indistinguishable from the predictions of the $\Lambda \mathrm{CDM}$ model. The same holds for the quartessence and, therefore, the total power spectrum.

It is important to remark that the above considered entropy perturbations are not isocurvature ones, since there are initial density perturbations, although no pressure fluctuations. Moreover, if one were to consider the evolution of the coupled baryonphoton fluid, we would set $\delta \rho_{b} / \dot{\rho}_{b}=\delta \rho_{q} / \dot{\rho}_{q}=(3 / 4) \delta \rho_{r} / \dot{\rho}_{r}$, which is sometimes referred 
in the literature as "adiabatic perturbations". Therefore, the entropy perturbations needed to eliminate the instabilities and oscillations in the power spectrum are not straightforwardly ruled out by cosmic microwave background radiation (CMB) observations, which have discarded purely isocurvature modes. A complete study of the CMB spectrum in quartessence models with entropy perturbations such as given by Eq. (19) remains to be done and would be an important test for the model.

\section{Discussion}

In what is currently considered the standard cosmological model, two exotic components, DM and DE, are invoked to explain two different phenomena: clustering of matter and cosmic acceleration. In spite of their success in explaining most of the cosmological observations, the exact nature of these two components remains a mystery. In fact, so far, it has not been proven that dark matter and dark energy are two distinct substances. This unsatisfactory situation stimulated the search for viable quartessence (unified-darkmatter) models [22, 23].

In this work we argue that adiabatic quartessence models with a convex equation of state always suffer from oscillations and suppression in the power spectrum, rendering those models inconsistent with the data. This can be understood in very simple terms, since, for that class of model, the maximum sound speed always occurs at the minimum density (at which $p=-\rho$ ). Therefore, the epoch of accelerated expansion is also a period of high adiabatic sound speed. This situation is different for equations of state that change concavity. In this case, the moment of maximum adiabatic sound speed can be disconnected from the start up of accelerated expansion. Such models are currently under investigation and may provide a power spectrum in agreement with the data.

Another alternative is to consider intrinsic entropy perturbations. In Ref. [11 it

was shown that, for QCM, if $\Gamma_{q}=-c_{s q}^{2} \delta_{q} / w_{q}$, or equivalently, that initial conditions are chosen such that $\delta p_{q}=0$, the oscillations and instabilities present in the adiabatic case, disappear. In this work, we have shown that the same type of entropy perturbations also solves the problem of the power spectrum for both the exponential and logarithmic quartessence models. This occurs because, in this case the effective sound speed vanishes $\left(c_{s e f f}^{2}=\delta p / \delta \rho=0\right)$, such that there is no gradient term in the "Euler equation" (17), and thus no oscillations and divergences appear in the power spectrum. We therefore argue that such entropy perturbations render generic quartessence models in agreement with power spectrum observations. However, although the nonadiabatic quartessence power spectrum is indistinguishable from that of "concordance" models like $\Lambda$ CDM, their behavior beyond the linear regime is quite different and, in principle, can be clearly distinguished with weak lensing observations [15].

To conclude, we remark that the quartessence scenario offers an alternative to concordance models, providing distinct predictions that can be tested with current data. Several dark energy models, being as "exotic" as quartessence, are nevertheless much more degenerate with the $\Lambda$ CDM model. It is therefore desirable to put some effort into 
deriving observational consequences of quartessence models.

Adiabatic convex quartessence now seem to be ruled out by the data. Nonadiabatic convex models might be discarded by weak lensing. However, models in which the equation of state changes its concavity are a promising alternative, even for adiabatic

perturbations [23]. Such models deserve to be further studied, offering potential alternatives for dark-matter and dark-energy unification. The search for quartessence models provides also a test for the robustness of the dark-matter plus dark-energy paradigm. If generic unifying-dark-matter models can be ruled out, this would give a strong support for the concordance cosmological models.

\section{Acknowledgments}

The authors would like to thank the following Brazilian research agencies for financial support: RRRR is partially supported by CAPES, MM is partially supported by FAPERJ and MCT, and IW is partially supported by CNPq. MM acknowledges the hospitality of Fermilab, where this manuscript was completed.

\section{References}

[1] For reviews, see, e.g., K. Griest, M. Kamionkowski, Phys. Rep. 333, 167-182 (2000); M.S. Turner, Phys. Rep. 197, 67 (1990); G.G. Raffelt, Phys. Rep. 198, 1 (1990).

[2] C. Wetterich, Nuclear Physics B302, 668 (1988); B. Ratra and P.J.E. Peebles, Phys. Rev. D37, 3406 (1988); J.A. Frieman, C.T. Hill, A. Stebbins, and I. Waga, Phys. Rev. Lett. 75, 2077 (1995); P. Ferreira, and M. Joyce, Phys. Rev. Lett. 79, 4740 (1997); R.R. Caldwell, R. Dave, and P.J. Steinhardt, Phys. Rev. Lett. 80, 1582 (1998); for reviews see, for instance, V. Sahni, and A.A. Starobinsky, Int. J. Mod. Phys. D 9, 373 (2000); S.M. Carrol, Living Review in Relativity 4, 1 (2001); and P.J.E. Peebles, and B. Ratra, Rev. Mod. Phys. 75, 559 (2003).

[3] M. Makler, S.Q. Oliveira, and I. Waga, Phys. Lett. B 555, 1, 0209486 (2003).

[4] A. Kamenshchik, U. Moschella, and V. Pasquier, Phys. Lett. B511, 265 (2001).

[5] M. Makler, Gravitational Dynamics of Structure Formation in the Universe, PhD Thesis, Brazilian Center for Research in Physics (2001).

[6] N. Bilić, G.B. Tupper, and R.D. Viollier, Phys. Lett. B535, 17 (2002).

[7] J.C. Fabris, S.V.B. Gonçalves, and P.E. Souza, Gen. Rel. Grav. 34, 53 (2002).

[8] M.C. Bento, O. Bertolami, and A.A. Sen, Phys. Rev. D66, 043507 (2002).

[9] M. Makler, S.Q. Oliveira, and I. Waga, Phys. Rev. D 64, 123521 (2003).

[10] H. Sandvik, M. Tegmark, M. Zaldarriaga, and I. Waga, Phys. Rev. D 69, 123524 (2004).

[11] R.R.R. Reis, I. Waga, M.O. Calvão, and S.E. Jorás, Phys. Rev. D 68, 061302(R) (2003).

[12] see, for instance, S.R. Cranmer - American Journal of Physics, in press (2004), astro-ph/0406176 (v1), and references therein.

[13] H. Kodama and M. Sasaki, Prog. Theor. Phys. Suppl. 78, 1 (1984).

[14] K. A. Malik, PhD thesis, University of Portsmouth, astro-ph/0101563

[15] R.R.R. Reis, M. Makler, and I. Waga, Phys. Rev. D 69, 101301(R) (2004).

[16] J.C. Fabris, S.V.B. Gonalves, R. de S Ribeiro, Gen. Rel. Grav. 36, 211 (2004).

[17] J. M. Bardeen, J. R. Bond, N. Kaiser, and A. S. Szalay, Astrophys. J. 304, 15 (1986).

[18] N. Sugiyama, Astrophys. J. Supp. 100, 281 (1995).

[19] L. M. G. Beça, P. P. Avelino, J. P. M. de Carvalho, and C. J. A. P. Martins, Phys. Rev. D 67, 101301(R) 2003. 
[20] M. Tegmark, et al. (the SDSS collaboration), Phys. Rev. D 69, 103501 (2004); M. Tegmark, et al. (the SDSS collaboration), Astrophys. J. 606, 702 (2004).

[21] M. Tegmark, A. J. S. Hamilton, and Y. Xu, Mon. Not. R. Astron. Soc. 335, 887 (2002).

[22] R. J. Scherrer, Phys. Rev. Lett. 93, 011301 (2004); L. P. Chimento, M. Forte, and R. Lazkoz, astro-ph/0407288 ; see also R. Mainini and S. A. Bonometto, Phys. Rev. Lett. 93, 121301 (2004) for a different approach.

[23] M. Makler, R.R.R. Reis, and I. Waga, in preparation. 